\title{
The Periods of RR Lyrae
}

\author{
Arthur N. Cox
}

Los Alamos Astrophysics

\begin{abstract}
RR Lyrae (0.566 day period) exhibits the Blasko effect that suggests another natural mode with almost the same period as the accepted fundamenta] radial mode. This mode might be nonradial, but no one has done an extensive evaluation of this idea. An investigation requires a model that includes the decp composition structure where g-modes of low angular (observable) degree have weight and amplitude. An RR Lyrae model including the outer half of the mass and more than $99 \%$ of the radius, based on an asymptotic giant branch model from Hollowell (private communication), see below, was used for this study. It includes composition gradient ramps between the primordial surface hydrogen and helium and the almost pure helium shell and the one between this helium shell and the convective core that is burning helium.

Nonradial mode periods almost resonant with the radial fundamental mode period seem to occur for all low $\ell$ values. In addition to significant pulsation amplitudes in the composition gradient regions where the Brunt Väisälä frequency is large, these low degree and low radial order modes have near-surface amplitudes very similar to the low order radial modes. These modes are evanescent in the convective core. Classical $\kappa$ and $\gamma$ effects give enough driving in the very low mass surface layers, so that important deep radiative damping for these modes does not completely stabilize nonradial g-mode pulsations. The $g_{4}, \ell=1$ mode gives a double-mode RR Lyrae with Blasko effect.

A nonradial mode may not always be visible, depending on how rotation presents the nonspherical pulsations to the observer. Thus the Blasko effect might come and go, as observed for maybe $20 \%$ of all RR Lyrae variables. For many, the Blasko effect may not be observable, even when a nonradial mode is there.
\end{abstract}

700 Mass Shell RR Lyrae Model

$\begin{array}{cc}\text { Mass }\left(M_{\odot}, \mathrm{g}\right) & 0.70,1.392 \times 10^{33} \\ \text { Effective Temperature }(\mathrm{K}) & 6800 \\ \text { Luminosity }(\mathrm{erg} / \mathrm{s}) & 2.272 \times 10^{35} \\ \text { Fundamental Mode Period }(\mathrm{d}) & 0.566 \\ \text { Growth Rate }(\Delta \mathrm{KE} / \mathrm{KE} \text { per period) } & 0.029 \\ g_{4} \text { Mode Period }(\mathrm{d}) & 0.570 \\ \text { Growth Rate }(\Delta \mathrm{KE} / \mathrm{KE} \text { per period) } & 0.0007 \\ \text { Blasko Period }(\mathrm{d}) & 81 \\ \text { Last Mass }(\mathrm{g}) & 2.53 \times 10^{24} \\ \text { Envelope Mass }(\mathrm{g}) & 6.96 \times 10^{32} \\ \text { Photosphere Radius }(\mathrm{cm}) & 3.862 \times 10^{11} \\ \text { Central Ball Radius }(\mathrm{cm}) & 4.536 \times 10^{9}\end{array}$

